ZMP-HH/09-8

Hamburger Beiträge zur Mathematik Nr. 330

\title{
Defects and Permutation branes in the Liouville field theory
}

\author{
Gor Sarkissian* \\ Organisationseinheit Mathematik, Universität Hamburg \\ Bereich Algebra und Zahlentheorie \\ Bundesstraße 55, D-20146 Hamburg
}

March 2009

\begin{abstract}
The defects and permutation branes for the Liouville field theory are considered. By exploiting cluster condition, equations satisfied by permutation branes and defects reflection amplitudes are obtained. It is shown that two types of solutions exist, discrete and continuous families.
\end{abstract}

\footnotetext{
* Email address:

sarkissian@math.uni-hamburg.de.
} 


\section{Introduction}

In this paper we address problem of construction permutation branes and topological defects in the Liouville field theory. Topological defects are defined as operators commuting with left and right copies of chiral algebra. In the last years they were studied extensively in RCFT see, e.g. [12, 13, 15, 20, 28, 29, 35, 39] and free bosonic theory $[1,2,16]$. There has been also progress in the Lagrangian description of the defects $[17,36,38]$.

In this paper we turn to construction of topological defects and closely related to them permutation branes in the Liouville field theory. A discussion of the topological defects in the Liouville theory can be found in [35]. It was conjectured there that defects in the Liouville theory should be labelled as FZZ and ZZ branes $[11,44]$ by the primaries and obey the corresponding fusion rules. Our findings here confirm this conjecture.

Main tool used in this paper is generalizations of the Cardy-Lewellen cluster condition to permutation branes and defects. In the past years Cardy-Lewellen sewing constraint proved to be very useful to find branes in non-rational models $[11,19,22,30,41,44]$. Here we show that for construction of defects in nonrational models it can serve as well. The paper is organized as follows. In section 2 permutation branes in RCFT are reviewed. We collected there necessary formulae for different annulus partition functions involving permutation branes. We also elaborate here Cardy-Lewellen cluster conditions for permutation branes. In section 3 defects in RCFT are reviewed. Here again a special attention to Cardy-Lewellen cluster condition for defects is paid. In section 4 we remind the necessary stuff on Liouville field theory. In section 5 permutation branes for Liouville theory are presented. In section 6 defects for Liouville theory are found.

\section{Permutation branes in RCFT}

Let us remind some basic facts on permutation branes in RCFT $[14,18,32,37]$. Consider $N$-fold tensor product of a CFT with chiral symmetry algebra $W_{L}\left(W_{R}\right)$.

On such a product one can consider brane with gluing automorphism given by a cycle $(1 \ldots N)$, or by other words, satisfying following equations:

$$
\begin{aligned}
& W_{L}^{(r)}(z)=\left.W_{R}^{(r+1)}(\bar{z})\right|_{z=\bar{z}}, \quad r=1 \ldots N-1 \\
& W_{L}^{(N)}(z)=\left.W_{R}^{(1)}(\bar{z})\right|_{z=\bar{z}}
\end{aligned}
$$


When single copy CFT is a rational CFT with diagonal partition function

$$
Z=\sum_{i, \bar{i}} Z_{i, \bar{i}} \chi_{i}(q) \bar{\chi}_{\bar{i}}(\bar{q}), \quad Z_{i, \bar{i}}=\delta_{i, i^{*}}, \quad q=\exp (2 i \pi \tau)
$$

where $i^{*}$ is conjugate representation in the sense $N_{i i^{*}}^{1}=1$, permutation branes were constructed in [32]. It is shown in [32] that for such a CFT permutation branes are labeled by primaries of single copy and have boundary states:

$$
\left.|a\rangle_{\mathcal{P}}=\sum_{j} \frac{S_{a j}}{\left(S_{0 j}\right)^{N / 2}}|j, j\rangle\right\rangle_{\mathcal{P}}
$$

where $S_{i j}$ is the matrix of the modular transformations of single copy:

$$
\chi_{i}(\tilde{q})=\sum_{j} S_{i j} \chi_{j}(q), \quad \tilde{q}=\exp (-2 i \pi / \tau)
$$

and $|j, j\rangle\rangle_{\mathcal{P}}$ permuted Ishibashi state satisfying (11). It is known that boundary states should satisfy two criteria: Cardy condition [6], requiring the annulus partition functions to be expressed as sum of some characters with non-negative integer numbers, and Cardy-Lewellen cluster condition [7,23]. It is shown in [32] that states (3) indeed satisfy the Cardy condition. In case of permutation branes check of the Cardy condition involves calculation of two kinds of annulus partition functions:

1) partition functions between two permutation branes,

2) partition function between permutation branes and factorized branes, i.e. branes which can be written as product of Cardy branes for each constituent.

For further use we write down these partition functions in case of two-fold product $N=2$. Generalization to generic $N$ is straightforward and corresponding formulae can be found in [32]. For two-fold product permutation boundary state (3) satisfies relations:

$$
\begin{array}{ll}
L_{n}^{(1)}-\bar{L}_{-n}^{(2)}=0, & W_{n}^{(1)}-(-1)^{s_{W}} \bar{W}_{-n}^{(2)}=0 \\
L_{n}^{(2)}-\bar{L}_{-n}^{(1)}=0, & W_{n}^{(2)}-(-1)^{s_{W}} \bar{W}_{-n}^{(1)}=0
\end{array}
$$

where $s_{W}$ is the spin of $W$, and takes form:

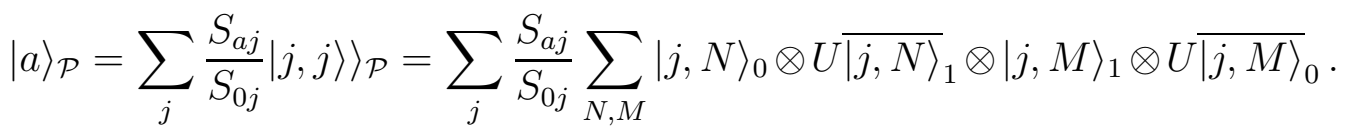

where 0 and 1 labels first and second copy of the CFT in question, sums over $N$ and $M$ run over orthonormal basis of the highest weight representation $R_{j}$, and 
operator $U$ in front of right-movers is chiral CPT operator as usual. Using this explicit expression, the Verlinde formula

$$
N_{i j}^{k}=\frac{S_{i l} S_{j l} S_{k l}^{*}}{S_{0 l}}
$$

and expression for the Cardy state:

$$
|i\rangle=\sum_{j} \frac{S_{i j}}{\sqrt{S_{0 j}}} \sum_{N}|j, N\rangle \otimes U \overline{|j, N\rangle}
$$

it is easy to compute that partition function between two permutation branes labeled by $a_{1}$ and $a_{2}$ is :

$$
Z_{a_{1}, a_{2}}=\sum_{r, k, l} N_{a_{1} r}^{a_{2}} N_{k l}^{r} \chi_{k}(q) \chi_{l}(q)
$$

and partition function between permutation brane labeled by $a$ and product of two Cardy states labeled by $a_{1}$ and $a_{2}$ respectively is ( for details see $[32,37]$ ):

$$
Z_{a,\left(a_{0} a_{1}\right)}=\sum_{k, r} N_{a_{0} a_{1}}^{r} N_{r k}^{a} \chi_{k}\left(q^{1 / 2}\right)
$$

Now we turn to the Cardy-Lewellen cluster condition [3,7,23,31,33,34]. Given that cluster condition for permutation branes very little discussed in the literature, we will derive it here for general case of the not necessarily diagonal RCFT with the arbitrary fusion coefficients $N_{i j}^{k}$. However to keep the things still enough simple we assume that we have no bulk multiplicities : $Z_{i \bar{i}}=0,1$.

Let us as warm-up exercise to remind cluster condition for usual branes. Consider a boundary state

$$
\left.|\alpha\rangle=\sum_{i} B_{\alpha}^{i}|i\rangle\right\rangle
$$

where $i$ runs over primaries, and $|i\rangle\rangle$ are Ishibashi states. Recall the relation between coefficients $B_{\alpha}^{i}$ and one-point functions

$$
\left\langle\Phi_{(i \bar{i})}(z, \bar{z})\right\rangle_{\alpha}=\frac{U_{\alpha}^{i} \delta_{i^{*} \bar{i}}}{(z-\bar{z})^{2 \Delta_{i}}}
$$

in the presence of the boundary condition $\alpha$ :

$$
U_{\alpha}^{i}=\frac{B_{\alpha}^{i}}{B_{\alpha}^{0}} e^{i \pi \Delta_{i}}
$$


It is convenient at this point to introduce full plane chiral decomposition of physical fields [26]:

$$
\Phi_{(i \bar{i})}(z, \bar{z})=\sum_{j, \bar{j}, k, \bar{k}, a, \bar{a}} C_{(i \bar{i})(j \bar{j}) a \bar{a}}^{(k \bar{k})}\left(\phi_{i j a}^{k}(z) \otimes \phi_{\overline{i j \bar{a}}}^{\bar{k}}(\bar{z})\right)
$$

where $\phi_{i j a}^{k}$ are intertwining operators $R_{j} \rightarrow R_{k}$, and $a=1 \ldots N_{i j}^{k}$. Consider now two-point function $\left\langle\Phi_{i}\left(z_{1}, \bar{z}_{1}\right) \Phi_{j}\left(z_{2}, \bar{z}_{2}\right)\right\rangle_{\alpha}$ in the presence of boundary in two pictures. In the first picture one applies first bulk OPE

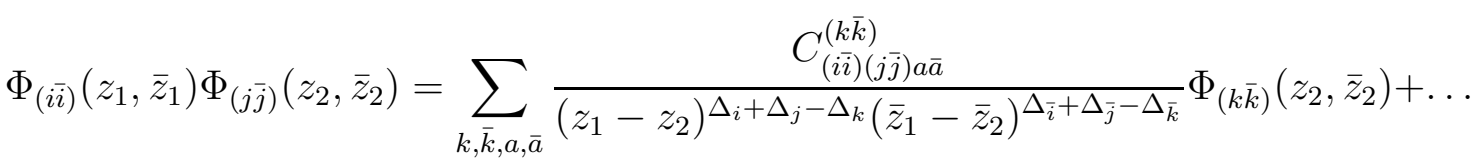

and then evaluates one-point function resulting in:

$$
\left\langle\Phi_{(i \bar{i})}\left(z_{1}, \bar{z}_{1}\right) \Phi_{(j \bar{j})}\left(z_{2}, \bar{z}_{2}\right)\right\rangle_{\alpha}=\sum_{k, a, \bar{a}} C_{(\bar{i} \bar{i})(j \bar{j}) a \bar{a}}^{\left(k, k^{*}\right)} U_{\alpha}^{k} \mathcal{T}_{k a \bar{a}}^{i j \bar{i} \bar{j}}
$$

where $\mathcal{T}_{k a \bar{a}}^{i j \bar{j}}$ are conformal blocks, which using $\phi_{i j a}^{k}$ intertwining operators can be expressed as:

$$
\mathcal{T}_{k a \bar{a}}^{i j \bar{i} \bar{j}}=\left\langle 0\left|\phi_{i i^{*}}^{1}\left(z_{1}\right) \phi_{j k^{*} a}^{i^{*}}\left(z_{2}\right) \phi_{\overline{i j} \bar{a}}^{k^{*}}\left(\bar{z}_{1}\right) \phi_{\bar{j} 1}^{\bar{j}}\left(\bar{z}_{2}\right)\right| 0\right\rangle
$$

In the second picture one first applies bulk-boundary OPE [3]

$$
\Phi_{(i \bar{i})}(z, \bar{z})=\sum_{m, t, s} \frac{R_{m, s,(\alpha)}^{(i \bar{i}), t}}{(z-\bar{z})^{\Delta_{i}+\Delta_{\bar{i}}-\Delta_{m}}} \psi_{m}^{\alpha \alpha, s}+\ldots
$$

where $t=1, \ldots N_{i \bar{i}}^{m}$, and index $s$ counts different boundary fields and runs $s=$ $1, \ldots n_{\alpha \alpha}^{m}$, where $n_{\alpha \alpha}^{m}$ coefficient of character $\chi_{m}$ in the annulus partition function between brane $\alpha$ with itself, and then evaluates two-point function of boundary fields resulting in

$$
\left\langle\Phi_{(i \bar{i})}\left(z_{1}, \bar{z}_{1}\right) \Phi_{(j \bar{j})}\left(z_{2}, \bar{z}_{2}\right)\right\rangle_{\alpha}=\sum_{m, t_{1}, t_{2}, s_{1}, s_{2}} R_{m, s_{1}(\alpha)}^{(\bar{i} \bar{i}), t_{1}} R_{m^{*}, s_{2}(\alpha)}^{(j \bar{j}), t_{2}} \mathcal{T}_{m t_{1} t_{2}}^{i \bar{i} \bar{j}} c_{m}^{\alpha, s_{1}, s_{2}}
$$

where

$$
\left\langle\psi_{m}^{\alpha \alpha, s_{1}}\left(x_{1}\right) \psi_{n}^{\alpha \alpha, s_{2}}\left(x_{2}\right)\right\rangle=\frac{c_{m}^{\alpha, s_{1}, s_{2}} \delta_{m n^{*}}}{\left|x_{2}-x_{1}\right|^{2 \Delta_{m}}}
$$

and

$$
\mathcal{T}_{m t_{1} t_{2}}^{i \bar{i} j \bar{j}}=\left\langle 0\left|\phi_{i i^{*}}^{1}\left(z_{1}\right) \phi_{\bar{i} m^{*} t_{1}}^{i^{*}}\left(\bar{z}_{1}\right) \phi_{j \bar{j} t_{2}}^{m^{*}}\left(z_{2}\right) \phi_{\bar{j} 1}^{\bar{j}}\left(\bar{z}_{2}\right)\right| 0\right\rangle
$$


Using braiding relations between chiral blocks

$$
\mathcal{T}_{k a \bar{a}}^{i j \bar{i} \bar{j}}=\sum_{m} B_{k^{*} m^{*}}^{(+)}\left[\begin{array}{cc}
j & \bar{i} \\
i^{*} & \bar{j}
\end{array}\right]_{a \bar{a}}^{t_{1} t_{2}} \mathcal{T}_{m t_{1} t_{2}}^{i \bar{i} j \bar{j}}
$$

one derives:

$$
\sum_{k, a, \bar{a}} C_{(i \bar{i})(j \bar{j}) a \bar{a}}^{\left(k, k^{*}\right)} U_{\alpha}^{k} B_{k^{*} m^{*}}^{(+)}\left[\begin{array}{cc}
j & \bar{i} \\
i^{*} & \bar{j}
\end{array}\right]_{a \bar{a}}^{t_{1} t_{2}}=\sum_{s_{1}, s_{2}} R_{m, s_{1},(\alpha)}^{(i \bar{i}), t_{1}} R_{m^{*}, s_{2},(\alpha)}^{(j \bar{j}) t_{2}} c_{m}^{\alpha, s_{1}, s_{2}}
$$

Putting $m=0$ one obtains:

$$
\sum_{k, a, \bar{a}} C_{\left(i i^{*}\right)\left(j j^{*}\right) a \bar{a}}^{\left(k, k^{*}\right)} U_{\alpha}^{k} B_{k^{*} 0}^{(+)}\left[\begin{array}{cc}
j & i^{*} \\
i^{*} & j^{*}
\end{array}\right]_{a \bar{a}}^{11}=U_{(\alpha)}^{i} U_{(\alpha)}^{j}
$$

where we took into account that $R_{0(\alpha)}^{i \bar{i}}=U_{\alpha}^{i} \delta_{i^{*} \bar{i}}$. We should note that here we used reflection amplitudes as they defined in [3]. The traditionally used reflection amplitudes $[7,23]$ differ by phase

$$
U_{(\alpha)}^{i}=\tilde{U}_{(\alpha)}^{i} e^{i \pi \Delta_{i}}
$$

They have the advantage, that related to boundary states coefficients without phase factor:

$$
\tilde{U}_{(\alpha)}^{i}=\frac{B_{\alpha}^{i}}{B_{\alpha}^{0}}
$$

Recalling relation between braiding and fusion matrices:

$$
B_{p q}^{(+)}\left[\begin{array}{cc}
i & j \\
k & l
\end{array}\right]_{a b}^{c d}=e^{i \pi\left(\Delta_{k}+\Delta_{l}-\Delta_{p}-\Delta_{q}\right)} F_{p q}\left[\begin{array}{cc}
i & l \\
k & j
\end{array}\right]_{a b}^{c d}
$$

and symmetry properties of fusion matrix

$$
F_{p q}\left[\begin{array}{cc}
k & j \\
i & l
\end{array}\right]_{a b}^{c d}=F_{p^{*} q^{*}}\left[\begin{array}{cc}
l & i^{*} \\
j^{*} & k
\end{array}\right]_{a b}^{c d}
$$

we receive that $\tilde{U}_{(\alpha)}^{i}$ obey the equation:

$$
\sum_{k, a, \bar{a}} C_{\left(i i^{*}\right)\left(j j^{*}\right) a \bar{a}}^{\left(k, k^{*}\right)} \tilde{U}_{\alpha}^{k} F_{k 0}\left[\begin{array}{cc}
i^{*} & i \\
j & j
\end{array}\right]_{a \bar{a}}^{11}=\tilde{U}_{(\alpha)}^{i} \tilde{U}_{(\alpha)}^{j}
$$

Now we apply this procedure to permutation branes. For simplicity we again consider the case of two-fold product. The primary fields of two-fold product are 
products of primary fields $\Phi_{i}^{(1)} \Phi_{j}^{(2)}$. The form of the gluing relations (15) implies that for permutation branes two-point functions have the form:

$$
\left\langle\Phi_{(i \bar{i})}^{(1)}\left(z_{1}\right) \Phi_{(j \bar{j})}^{(2)}\left(z_{2}\right)\right\rangle_{\mathcal{P}}=\frac{U_{(\mathcal{P})}^{i, \bar{i}} \delta_{i \bar{j}^{*}} \delta_{\bar{i} j^{*}}}{\left(z_{1}-\bar{z}_{2}\right)^{2 \Delta_{i}\left(\bar{z}_{1}-z_{2}\right)^{2 \Delta_{\bar{i}}}}}
$$

To receive cluster condition for permutation branes one should consider four-point functions $\left\langle\Phi_{\left(i_{1} \bar{i}_{1}\right)}^{(1)}\left(z_{1}\right) \Phi_{\left(i_{2} \bar{i}_{2}\right)}^{(2)}\left(z_{2}\right) \Phi_{\left(j_{1} \bar{j}_{1}\right)}^{(1)}\left(z_{3}\right) \Phi_{\left(j_{2} \bar{j}_{2}\right)}^{(2)}\left(z_{4}\right)\right\rangle_{\mathcal{P}}$. In the first picture one has:

$$
\begin{aligned}
& \left\langle\Phi_{\left(i_{1} \bar{i}_{1}\right)}^{(1)}\left(z_{1}\right) \Phi_{\left(i_{2} \bar{i}_{2}\right)}^{(2)}\left(z_{2}\right) \Phi_{\left(j_{1} \bar{j}_{1}\right)}^{(1)}\left(z_{3}\right) \Phi_{\left(j_{2} \bar{j}_{2}\right)}^{(2)}\left(z_{4}\right)\right\rangle_{\mathcal{P}}= \\
& \sum_{k, \bar{k}, a, \bar{a}, c, \bar{c}} C_{\left(i_{1} \bar{i}_{1}\right)\left(j_{1} \bar{j}_{1}\right) a \bar{a}}^{(k, \bar{a}} C_{\left(i_{2} \bar{i}_{2}\right)\left(j_{2} \bar{j}_{2}\right) c \bar{c}}^{\left(\bar{k}^{*}, k^{*}\right)} U_{(\mathcal{P})}^{k, \bar{k}} \mathcal{M}_{k \bar{k} a \bar{a} c \bar{c}}^{i_{1} i_{2} j_{1} j_{2} \bar{i}_{1} \bar{i}_{2} \bar{j}_{1} \bar{j}_{2}}
\end{aligned}
$$

where $\mathcal{M}_{k \bar{k} a \bar{a} c \bar{c}}^{i_{1} i_{2} j_{1} j_{2} \bar{i}_{1} \bar{i}_{2} \bar{j}_{1} \bar{j}_{2}}$ have the same form as $\mathcal{T}_{k}^{i j i \bar{j}}$ but with every field being product of two fields for each copy. Remembering gluing conditions (15) we note that actually left fields of the first copy related only to right fields of the second copy, and right field of the first copy to the left field of the second. Therefore $\mathcal{M}_{k \bar{k} a \bar{a} c \bar{c}}^{i_{1} i_{2} j_{1} j_{2} \bar{i}_{1} \bar{i}_{2} \bar{j}_{1} \bar{j}_{2}}$ factorize and have the form:

$$
\begin{aligned}
& \mathcal{M}_{k \bar{k} a \bar{a} c \bar{c}}^{i_{1} i_{2} j_{1} \bar{i}_{2} \bar{i}_{1} \bar{i}_{2} \bar{j}_{1} \bar{j}_{2}}= \\
& \left\langle 0\left|\phi_{i_{1} i_{1}^{*}}^{1}\left(z_{1}\right) \phi_{j_{1} k^{*} a}^{i_{*}^{*}}\left(z_{3}\right) \phi_{\bar{i}_{2} \bar{j}_{2} \bar{c}}^{k^{*}}\left(\bar{z}_{2}\right) \phi_{\bar{j}_{2} 1}^{\bar{j}_{2}}\left(\bar{z}_{4}\right)\right| 0\right\rangle \times \\
& \left\langle 0\left|\phi_{i_{2} i_{2}^{*}}^{1}\left(z_{2}\right) \phi_{j_{2} \bar{k}_{c}}^{i_{2}^{*}}\left(z_{4}\right) \phi_{\bar{i}_{1} \bar{j}_{1} \bar{a}}^{\bar{k}}\left(\bar{z}_{1}\right) \phi_{\bar{j}_{1} 1}^{\bar{j}_{1}}\left(\bar{z}_{3}\right)\right| 0\right\rangle=\mathcal{T}_{k a \bar{c}}^{i_{1} j_{1} \bar{i}_{2} \bar{j}_{2}} \mathcal{T}_{\bar{k}^{*} c \bar{a}}^{i_{2} j_{2} \bar{i}_{1} \bar{j}_{1}}
\end{aligned}
$$

Boundary OPE now looks:

$$
\Phi_{\left(i_{1} \bar{i}_{1}\right)}^{(1)}\left(z_{1}\right) \Phi_{\left(i_{2} \bar{i}_{2}\right)}^{(2)}\left(z_{2}\right)=\sum_{m n, t_{1}, t_{2}, s} \frac{R_{m n, s}^{\left(i_{1} \bar{i}_{1}\right),\left(i_{2} \bar{i}_{2}\right), t_{1}, t_{2}}}{\left(z_{1}-\bar{z}_{2}\right)^{\Delta_{i_{1}}+\Delta_{\bar{i}_{2}}-\Delta_{m}}\left(\bar{z}_{1}-z_{2}\right)^{\Delta_{\bar{i}_{1}}+\Delta_{i_{2}}-\Delta_{n}}} \psi_{m n}^{s}+\ldots
$$

where $t_{1}=1 \ldots N_{i_{1} \bar{i}_{2}}^{m}, t_{2}=1 \ldots N_{\bar{i}_{1} i_{2}}^{n}$, and $s$ counts different boundary fields, and its range is given by the corresponding coefficient in the annulus partition function of the permutation brane with itself. Using (33) in the second picture one has:

$$
\begin{aligned}
& \left\langle\Phi_{\left(i_{1} \bar{i}_{1}\right)}^{(1)}\left(z_{1}\right) \Phi_{\left(i_{2} \bar{i}_{2}\right)}^{(2)}\left(z_{2}\right) \Phi_{\left(j_{1} \bar{j}_{1}\right)}^{(1)}\left(z_{3}\right) \Phi_{\left(j_{2} \bar{j}_{2}\right)}^{(2)}\left(z_{4}\right)\right\rangle_{\mathcal{P}}= \\
& \sum_{m, n, t_{1}, t_{2}, t_{3}, t_{4}, s_{1}, s_{2}} R_{m n, s_{1}}^{\left(i_{1} \bar{i}_{1}\right),\left(i_{2} \bar{i}_{2}\right), t_{1}, t_{2}} R_{m^{*} n^{*}, s_{2}}^{\left(j_{1} \bar{j}_{1}\right),\left(j_{2} \bar{j}_{2}\right), t_{3}, t_{4}} c_{m n}^{s_{1}, s_{2}} \mathcal{M}_{m n t_{1} t_{2} t_{3} t_{4}}^{i_{1} i_{2} \bar{i}_{1} \bar{i}_{2} j_{1} j_{2} \bar{j}_{1} \bar{j}_{2}}
\end{aligned}
$$

where

$$
\left\langle\psi_{m n}^{s}\left(x_{1}\right) \psi_{p t}^{s}\left(x_{2}\right)\right\rangle=\frac{c_{m n}^{s_{1}, s_{2}} \delta_{m p^{*}} \delta_{n t^{*}}}{\left|x_{1}-x_{2}\right|^{2\left(\Delta_{m}+\Delta_{n}\right)}}
$$


and

$$
\begin{aligned}
& \mathcal{M}_{m n t_{1} t_{2} t_{3} t_{4}}^{i_{1} i_{2} \bar{i}_{1} \bar{i}_{2} j_{1} \bar{j}_{1} \bar{j}_{1} \bar{j}_{2}}= \\
& \left\langle 0\left|\phi_{i_{1} i_{1}^{*}}^{1}\left(z_{1}\right) \phi_{\bar{i}_{2} m^{*} t_{1}}^{i_{*}^{*}}\left(\bar{z}_{2}\right) \phi_{j_{1} \bar{j}_{2} t_{3}}^{m^{*}}\left(z_{3}\right) \phi_{\bar{j}_{2} 1}^{\bar{j}_{2}}\left(\bar{z}_{4}\right)\right| 0\right\rangle \times \\
& \left\langle 0\left|\phi_{i_{2} i_{2}^{*}}^{1}\left(z_{2}\right) \phi_{\bar{i}_{1} n^{*} t_{2}}^{i_{*}^{*}}\left(\bar{z}_{1}\right) \phi_{j_{2} \bar{j}_{1} t_{4}}^{n_{*}^{*}}\left(z_{4}\right) \phi_{\bar{j}_{1} 1}^{\bar{j}_{1}}\left(\bar{z}_{3}\right)\right| 0\right\rangle=\mathcal{T}_{m t_{1} t_{3}}^{i_{1} \bar{i}_{2} j_{1} \bar{j}_{2}} \mathcal{T}_{n t_{2} t_{4}}^{i_{2} \bar{i}_{1} j_{2} \bar{j}_{1}}
\end{aligned}
$$

Using (22) we end up with:

$$
\begin{aligned}
\sum_{k, \bar{k}, a, \bar{a}, c, \bar{c}} C_{\left(i_{1} \bar{i}_{1}\right)\left(j_{1} \bar{j}_{1}\right) a \bar{a}}^{(k, \bar{a}} C_{\left(i_{2}, \bar{i}_{2}\right)\left(j_{2} \bar{j}_{2}\right) c \bar{c}}^{\left(\bar{k}^{*}, k^{*}\right)} B_{k^{*} m^{*}}^{(+)} & {\left[\begin{array}{cc}
j_{1} & \bar{i}_{2} \\
i_{1}^{*} & \bar{j}_{2}
\end{array}\right]_{a \bar{c}}^{t_{1} t_{3}} B_{\bar{k} n^{*}}^{(+)}\left[\begin{array}{cc}
j_{2} & \bar{i}_{1} \\
i_{2}^{*} & \bar{j}_{1}
\end{array}\right]_{c \bar{a}}^{t_{2} t_{4}} U_{(\mathcal{P})}^{k, \bar{k}}=(37) } \\
& \sum_{s_{1}, s_{2}} R_{m n, s_{1}}^{\left(i_{1} \bar{i}_{1}\right),\left(i_{2} \bar{i}_{2}\right), t_{1}, t_{2}} R_{m^{*} n^{*}, s_{2}}^{\left(j_{1} \bar{j}_{1}\right),\left(j_{2} \bar{j}_{2}\right), t_{3}, t_{4}} c_{m n}^{s_{1}, s_{2}}
\end{aligned}
$$

Putting $m=n=0$, and taking into account that

$$
R_{00, s}^{\left(i_{1} \bar{i}_{1}\right),\left(i_{2} \bar{i}_{2}\right), t_{1}, t_{2}}=U_{(\mathcal{P})}^{i_{1}, \bar{i}_{1}} \delta_{i_{1}^{*} \bar{i}_{2}} \delta_{i_{2} \bar{i}_{1}^{*}}
$$

one obtains:

$$
\begin{array}{r}
\sum_{k, \bar{k}, a, \bar{a}, c, \bar{c}} C_{\left(i_{1} \bar{i}_{1}\right)\left(j_{1} \bar{j}_{1}\right) a \bar{a}}^{(k, \bar{k}} C_{\left(\bar{i}_{1}^{*} i_{1}^{*}\right)\left(\bar{j}_{1}^{*} j_{1}^{*}\right) c \bar{c}}^{\left(\bar{k}^{*}, k^{*}\right)} B_{k^{*} 0}^{(+)}\left[\begin{array}{cc}
j_{1} & i_{1}^{*} \\
i_{1}^{*} & j_{1}^{*}
\end{array}\right]_{a \bar{c}}^{11} B_{\bar{k} 0}^{(+)}\left[\begin{array}{cc}
\bar{j}_{1}^{*} & \bar{i}_{1} \\
\bar{i}_{1} & \bar{j}_{1}
\end{array}\right]_{c \bar{a}}^{11} U_{(\mathcal{P})}^{k, \bar{k}}= \\
U_{(\mathcal{P})}^{i_{1}, \bar{i}_{1}} U_{(\mathcal{P})}^{j_{1}, \bar{j}_{1}}
\end{array}
$$

Again defining new amplitudes

$$
\tilde{U}_{(\mathcal{P})}^{i_{1}, \overline{,}_{1}}=U_{(\mathcal{P})}^{i_{1}, \overline{,}_{1}} e^{i \pi\left(\Delta_{i}+\Delta_{\bar{i}}\right)}
$$

and using (27) and (28) we derive:

$$
\sum_{k, \bar{k}, a, \bar{a}, c, \bar{c}} C_{\left(i_{1} \bar{i}_{1}\right)\left(j_{1} \bar{j}_{1}\right) a \bar{a}}^{(k, \bar{k})} C_{\left(\bar{i}_{1}^{*} i_{1}^{*}\right)\left(\bar{j}_{1}^{*} j_{1}^{*}\right) c \bar{c}}^{\left(\bar{k}^{*}, k^{*}\right)} F_{k 0}\left[\begin{array}{cc}
i_{1}^{*} & i_{1} \\
j_{1}^{*} & j_{1}
\end{array}\right]_{a \bar{c}}^{11} F_{\bar{k}^{*} 0}\left[\begin{array}{cc}
\bar{i}_{1} & \bar{i}_{1}^{*} \\
\bar{j}_{1}^{*} & \bar{j}_{1}^{*}
\end{array}\right]_{c \bar{a}}^{11} \tilde{U}_{(\mathcal{P})}^{k, \bar{k}}=
$$

For diagonal model $i_{1}=\bar{i}_{1}^{*}, j_{1}=\bar{j}_{1}^{*}, \bar{k}=k^{*}$ without multiplicities $N_{i j}^{k}=1$, (41) simplifies to

$$
\sum_{k}\left(C_{i j}^{k}\right)^{2} \tilde{U}_{(\mathcal{P})}^{k}\left(F_{k 0}\left[\begin{array}{ll}
i^{*} & i \\
j & j
\end{array}\right]\right)^{2}=\tilde{U}_{(\mathcal{P})}^{i} \tilde{U}_{(\mathcal{P})}^{j}
$$


were we denoted $C_{i j}^{k} \equiv C_{\left(i i^{*}\right)\left(j j^{*}\right)}^{\left(k k^{*}\right)}$ and $\tilde{U}_{(\mathcal{P})}^{i} \equiv \tilde{U}_{(\mathcal{P})}^{i, i^{*}}$.

Note that for diagonal models permutation branes reflection amplitudes depend only on single copy primaries.

For this case permutation branes cluster condition was discussed in [32].

It is straightforward to generalize (41) to general $N$-fold product. Here we only write the corresponding formula for diagonal models (2) without multiplicities:

$$
\sum_{k}\left(C_{i j}^{k}\right)^{N} \tilde{U}_{(\mathcal{P})}^{k}\left(F_{k 0}\left[\begin{array}{ll}
i^{*} & i \\
j & j
\end{array}\right]\right)^{N}=\tilde{U}_{(\mathcal{P})}^{i} \tilde{U}_{(\mathcal{P})}^{j}
$$

It is shown in [32] that (3) satisfies (43).

\section{Topological defects in RCFT}

Recall basic facts on topological defects in RCFT $[15,20,28,29]$. The construction of defects lines is analogous to that of boundary condition. Following [28] we define defect lines as operators $X$, satisfying relations:

$$
\begin{gathered}
{\left[L_{n}, X\right]=\left[\bar{L}_{n}, X\right]=0} \\
{\left[W_{n}, X\right]=\left[\bar{W}_{n}, X\right]=0}
\end{gathered}
$$

As in the case of the boundary conditions, there are also consistency conditions, analogous to the Cardy and Cardy-Lewellen constraints, which must be satisfied by the operator $X$. For simplicity we shall write all the formulae for diagonal models (2). To formulate these conditions, one first note that as consequence of (44) and (45) $X$ is a sum of projectors

$$
X=\sum_{i, \bar{i}} \mathcal{D}^{(i, \bar{i})} P^{(i, \bar{i})}
$$

where

$$
P^{(i, \bar{i})}=\sum_{N, \bar{N}}(|i, N\rangle \otimes|\bar{i}, \bar{N}\rangle)(\langle i, N| \otimes\langle\bar{i}, \bar{N}|)
$$

An analogue of the Cardy condition for defects requires that partition function with insertion of a pair defects after modular transformation can be expressed as sum of characters with non-negative integers. It is found in [28] that for diagonal models one can solve this condition taking for each primary $a$

$$
\mathcal{D}_{a}^{(i, \bar{i})}=\frac{S_{a i}}{S_{0 i}}
$$


for which one has:

$$
Z_{a b}=\operatorname{Tr}\left(X_{a}^{\dagger} X_{b} \tilde{q}^{L_{0}-\frac{c}{24}} \tilde{\bar{q}}^{\bar{L}_{0}-\frac{c}{24}}\right)=\sum_{k, i \bar{i}} N_{b k}^{a} N_{i \bar{i}}^{k} \chi_{i}(q) \chi_{\bar{i}}(\bar{q})
$$

Topological defects can act on boundary states producing new boundary states. The action of defects (48) on Cardy states is easily obtained using the Verlinde formula:

$$
X_{a}|b\rangle=\sum_{d} N_{a b}^{d}|d\rangle
$$

Topological defects can be fused. For defects (48) again using the Verlinde formula one derives:

$$
X_{a} X_{b}=\sum_{c} N_{a b}^{c} X_{c}
$$

Now we turn to the cluster condition for defects [29]. Here we should consider two-point functions

$$
\begin{aligned}
& \left\langle\Phi_{i^{*}}\left(z_{1}, \bar{z}_{1}\right) X \Phi_{i}\left(z_{2}, \bar{z}_{2}\right)\right\rangle=\frac{D^{(i, \bar{i})}}{\left(z_{1}-z_{2}\right)^{2 \Delta_{i}\left(\bar{z}_{1}-\bar{z}_{2}\right)^{2 \Delta_{\bar{i}}}}} \\
& D^{i}=\frac{\mathcal{D}^{(i, \bar{i})}}{\mathcal{D}^{0}}
\end{aligned}
$$

Using (14) one can write for the following four-point function with the defects insertion in the first picture:

$$
\begin{aligned}
& \left\langle\Phi_{j^{*}}\left(z_{1}, \bar{z}_{1}\right) \Phi_{i^{*}}\left(z_{2}, \bar{z}_{2}\right) X \Phi_{i}\left(z_{3}, \bar{z}_{3}\right) \Phi_{j}\left(z_{4}, \bar{z}_{4}\right) X^{\dagger}\right\rangle= \\
& \sum_{k} C_{j^{*} j}^{1} C_{i j, a \bar{a}}^{k} C_{i^{*} k, c \bar{c}}^{j} D^{(k, \bar{k})} \mathcal{F}_{k a c}^{j^{*} i^{*} i j} \mathcal{F}_{\bar{k} \bar{a} \bar{c}}^{\bar{j}^{*} \bar{i}^{*} \bar{i}}
\end{aligned}
$$

where

$$
\mathcal{F}_{k a c}^{j^{*} i^{*} i j}=\left\langle 0\left|\phi_{j^{*} j}^{1}\left(z_{1}\right) \phi_{i^{*} k c}^{j}\left(z_{2}\right) \phi_{i j a}^{k}\left(z_{3}\right) \phi_{j 1}^{j}\left(z_{4}\right)\right| 0\right\rangle
$$

Here we denoted $C_{i j}^{k} \equiv C_{\left(i i^{*}\right)\left(j j^{*}\right)}^{\left(k k^{*}\right)}$ as before.

Using relations:

$$
C_{i^{*} k, c \bar{c}}^{j}=C_{k i^{*}, c \bar{c}}^{j}
$$

and

$$
C_{k i^{*}, c \bar{c}}^{j} C_{j^{*} j}^{1}=C_{i^{*} j^{*}, c \bar{c}}^{k^{*}} C_{k^{*} k}^{1}
$$

we can write for the second line of (54)

$$
\sum_{k} C_{i j, a \bar{a}}^{k} C_{i^{*} j^{*}, c \bar{c}}^{k^{*}} C_{k^{*} k}^{1} D^{(k, \bar{k})} \mathcal{F}_{k a c}^{j^{*} i^{*} i j} \mathcal{F}_{\bar{k} \bar{a} \bar{c}}^{\overline{j^{*}} \bar{i}^{*} \bar{i} \bar{j}}
$$


In the second picture one has:

$$
\begin{array}{r}
\left\langle\Phi_{i^{*}}\left(z_{2}, \bar{z}_{2}\right) X \Phi_{i}\left(z_{3}, \bar{z}_{3}\right) \Phi_{j}\left(z_{4}, \bar{z}_{4}\right) X^{\dagger} \Phi_{j^{*}}\left(z_{1}, \bar{z}_{1}\right)\right\rangle= \\
C_{i^{*} i}^{1} C_{j^{*} j}^{1} D^{(i, \bar{i})} D^{(j, \bar{j})} \mathcal{F}_{0}^{i^{*} i j j^{*}} \mathcal{F}_{0}^{\bar{i}^{*} \bar{i} \bar{j} \bar{j}^{*}}+\ldots
\end{array}
$$

where

$$
\mathcal{F}_{p m n}^{i^{*} i j j^{*}}=\left\langle 0\left|\phi_{i^{*} i}^{1}\left(z_{2}\right) \phi_{i p m}^{i}\left(z_{3}\right) \phi_{j j^{*} n}^{p}\left(z_{4}\right) \phi_{j^{* 1}}^{j^{*}}\left(z_{1}\right)\right| 0\right\rangle
$$

To relate (54) with (59) one should use braiding relations for chiral blocks to move $j^{*}$ to the very right. Using (27) and the following property of the braiding matrix

$$
B_{i j}^{(+)}\left[\begin{array}{cc}
i^{*} & j^{*} \\
0 & k
\end{array}\right]_{1 a}^{1 a}=( \pm) e^{i \pi\left(\Delta_{k}-\Delta_{i}-\Delta_{j}\right)}
$$

one obtains product of fusion matrices :

$$
\mathcal{F}_{k a c}^{j^{*} i^{*} i j} \mathcal{F}_{\bar{k} \bar{a} \bar{c}}^{\bar{j}^{*} \bar{i}^{*} \overline{i j}}=F_{k 0}\left[\begin{array}{cc}
j^{*} & j \\
i & i
\end{array}\right]_{a c}^{11} F_{\bar{k} 0}\left[\begin{array}{cc}
\bar{j}^{*} & \bar{j} \\
\bar{i} & \bar{i}
\end{array}\right]_{\bar{a} \bar{c}}^{11} \mathcal{F}_{0}^{i^{*} i j j^{*}} \mathcal{F}_{0}^{\bar{i}^{*} \bar{i} \bar{j} \bar{j}^{*}}+\ldots
$$

Collecting all we obtain

$$
\begin{array}{r}
\sum_{k}\left(C_{k^{*} k}^{1} D^{(k \bar{k})}\right) C_{i j, a \bar{a}}^{k} C_{i^{*} j^{*}, c \bar{c}}^{k^{*}} F_{k 0}\left[\begin{array}{cc}
j^{*} & j \\
i & i
\end{array}\right]_{a c}^{11} F_{\bar{k} 0}\left[\begin{array}{cc}
\bar{j}^{*} & \bar{j} \\
\bar{i} & \bar{i}
\end{array}\right]_{\bar{a} \bar{c}}^{11}= \\
\left(C_{i^{*} i}^{1} D^{(i \bar{i})}\right)\left(C_{\left(j^{*} j\right.}^{1} D^{(j \bar{j})}\right)
\end{array}
$$

Comparing formulae (15) and (44), (6) and (46), (48), (41) and (63) one reveals deep connection between permutation branes on two-fold product form one side, and defects on other side, known as folding trick $[1,2,27,43]$. We see that mentioned relations for permutation branes become corresponding relations for defect after performing two-steps operation (folding) on the second copy of the CFT in question: left-right exchange and then hermitian conjugation, turning boundary state to operator. Comparison of (41) and (63) shows that the hermitian conjugation requires inclusion of the two-point functions $C_{i^{*} i}^{1}$.

\section{Liouville theory}

Let us review basic facts on the Liouville field theory (see e.g. [42]). Liouville field theory is defined on a two-dimensional surface with metric $g_{a b}$ by the local 
Lagrangian density

$$
\mathcal{L}=\frac{1}{4 \pi} g_{a b} \partial_{a} \varphi \partial_{b} \varphi+\mu e^{2 b \varphi}+\frac{Q}{4 \pi} R \varphi
$$

where $R$ is associated curvature. This theory is conformal invariant if the coupling constant $b$ is related with the background charge $Q$ as

$$
Q=b+\frac{1}{b}
$$

The symmetry algebra of this conformal field theory is the Virasoro algebra

$$
\left[L_{m}, L_{n}\right]=(m-n) L_{m+n}+\frac{c_{L}}{12}\left(n^{3}-n\right) \delta_{n,-m}
$$

with central charge

$$
c_{L}=1+6 Q^{2}
$$

Primary fields $V_{\alpha}$ in this theory, which are associated with exponential fields $e^{2 \alpha \varphi}$, have conformal dimensions

$$
\Delta_{\alpha}=\alpha(Q-\alpha)
$$

The fields $V_{\alpha}$ and $V_{Q-\alpha}$ have the same conformal dimensions and represent the same primary field, i.e. they are proportional to each other:

$$
V_{\alpha}=S(\alpha) V_{Q-\alpha}
$$

with the function

$$
S(\alpha)=\frac{\left(\pi \mu \gamma\left(b^{2}\right)\right)^{b^{-1}(Q-2 \alpha)}}{b^{2}} \frac{\Gamma(1-b(Q-2 \alpha)) \Gamma\left(-b^{-1}(Q-2 \alpha)\right)}{\Gamma(b(Q-2 \alpha)) \Gamma\left(1+b^{-1}(Q-2 \alpha)\right)}
$$

The spectrum of the Liouville theory is believed $[4,5,8]$ to be of the following form

$$
\mathcal{H}=\int_{0}^{\infty} d p R_{\frac{Q}{2}+i P} \otimes R_{\frac{Q}{2}+i P}
$$

where $R_{\alpha}$ is the highest weight representation with respect to Virasoro alegbra. Characters of the representations $R_{\frac{Q}{2}+i P}$ are

$$
\chi_{P}(\tau)=\frac{q^{P^{2}}}{\eta(\tau)}
$$

where

$$
\eta(\tau)=q^{1 / 24} \prod_{n=1}^{\infty}\left(1-q^{n}\right)
$$


Modular transformation of (72) is well-known:

$$
\chi_{P}\left(-\frac{1}{\tau}\right)=\sqrt{2} \int \chi_{P^{\prime}}(\tau) e^{4 i \pi P P^{\prime}} d P^{\prime}
$$

Degenerate representations appear at $\alpha_{m, n}=\frac{1-m}{2 b}+\frac{1-n}{2} b$ and have conformal dimensions [21]

$$
\Delta_{m, n}=Q^{2} / 4-(m / b+n b)^{2} / 4
$$

where $m, n$ are positive integers. At general $b$ there is only one null-vector at the level $m n$. Hence the degenerate character reads:

$$
\chi_{m, n}(\tau)=\frac{q^{-(m / b+n b)^{2}}-q^{-(m / b-n b)^{2}}}{\eta(\tau)}
$$

Modular transformation of (76) is worked out in [44]

$$
\chi_{m, n}\left(-\frac{1}{\tau}\right)=2 \sqrt{2} \int \chi_{P}(\tau) \sinh (2 \pi m P / b) \sinh (2 \pi n b P) d P
$$

For future use we write here the reflection function for $\alpha=\frac{Q}{2}+i P$, denoting it briefly as $S(P)$ :

$$
S(P)=-\left[\pi \mu \gamma\left(b^{2}\right)\right]^{-i 2 P / b} \frac{\Gamma(1+2 i b P) \Gamma\left(1+\frac{2 i P}{b}\right)}{\Gamma(1-2 i b P) \Gamma\left(1-\frac{2 i P}{b}\right)}
$$

Two-point functions of Liouvulle theory are given by reflection function (70):

$$
\left\langle V_{\alpha}\left(z_{1}, \bar{z}_{1}\right) V_{\alpha}\left(z_{2}, \bar{z}_{2}\right)\right\rangle=\frac{S(\alpha)}{\left(z_{1}-z_{2}\right)^{2 \Delta_{\alpha}}\left(\bar{z}_{1}-\bar{z}_{2}\right)^{2 \Delta_{\alpha}}}
$$

Three-point functions of Liouville theory $C\left(\alpha_{1}, \alpha_{2}, \alpha_{3}\right)$ are computed in [9, 45], were so called DOZZ formula for them was suggested. We don't need in this paper the full DOZZ formula. But we do need $C\left(\alpha_{1}, \alpha_{2}, \alpha_{3}\right)$ for the values of $\alpha_{i}$ satisfying relation

$$
\alpha_{1}+\alpha_{2}+\alpha_{3}=Q-n b
$$

For this case three-point functions are given by the screening integrals computed in $[10]$

$$
I_{n}\left(\alpha_{1}, \alpha_{2}, \alpha_{3}\right)=\left(b^{4} \gamma\left(b^{2}\right) \pi \mu\right)^{n} \frac{\prod_{j=1}^{n} \gamma\left(-j b^{2}\right)}{\prod_{k=0}^{n-1}\left[\gamma\left(2 \alpha_{1} b+k b^{2}\right) \gamma\left(2 \alpha_{2} b+k b^{2}\right) \gamma\left(2 \alpha_{3} b+k b^{2}\right)\right]}
$$

where $\gamma(x)=\frac{\Gamma(x)}{\Gamma(1-x)}$.

Structure constants $C_{\alpha_{1}, \alpha_{2}}^{\alpha_{3}}$ are related to three-point functions as

$$
C_{\alpha_{1}, \alpha_{2}}^{\alpha_{3}}=C\left(\alpha_{1}, \alpha_{2}, Q-\alpha_{3}\right)
$$




\section{Permutation branes in Liouville theory}

In this section we turn to construction of permutation branes on $N$-fold product of the Liouville field theories. As explained in section 1 they satisfy following gluing conditions:

$$
\begin{aligned}
& L_{n}^{(r)}-\bar{L}_{-n}^{(r)}=0, \quad r=1 \ldots N-1, \\
& L_{n}^{(N)}-\bar{L}_{-n}^{(1)}=0 .
\end{aligned}
$$

Remembering that Liouville field theory is diagonal theory (71) we conclude that reflection amplitudes as well as Ishibashi states depend on single copy primaries $P$. To compute reflection amplitudes $U_{\mathcal{P}}^{(N)}\left(\alpha=\frac{Q}{2}+i P\right)$ for permutation branes on $N$-fold product of Liouville fields

$$
\left\langle V_{\frac{Q}{2}+i P}^{(1)}\left(z_{1}, \bar{z}_{1}\right) \cdots V_{\frac{Q}{2}+i P}^{(N)}\left(z_{N}, \bar{z}_{N}\right)\right\rangle_{\mathcal{P}}=\frac{U_{\mathcal{P}}^{(N)}(\alpha)}{\prod_{1}^{N}\left(z_{r}-\bar{z}_{r+1}\right)^{\left(Q^{2} / 2+2 P^{2}\right)}}
$$

where $z_{N+1} \equiv z_{1}$, we will use the same trick as in $[11,40,44]$ and apply sewing constraints to $2 N$-point function

$$
\left\langle V_{-b / 2}^{(1)}\left(z_{1}, \bar{z}_{1}\right) \cdots V_{-b / 2}^{(N)}\left(z_{N}, \bar{z}_{N}\right) V_{\frac{Q}{2}+i P}^{(1)}\left(z_{N+1}, \bar{z}_{N+1}\right) \cdots V_{\frac{Q}{2}+i P}^{(N)}\left(z_{2 N}, \bar{z}_{2 N}\right)\right\rangle_{\mathcal{P}}
$$

with degenerate representation $-b / 2$. Recalling fusion rule with degenerate field

$$
V_{-b / 2} V_{\alpha} \sim C_{-b / 2, \alpha}^{\alpha-b / 2} V_{\alpha-b / 2}+C_{-b / 2, \alpha}^{\alpha+b / 2} V_{\alpha+b / 2}
$$

and that Liouville theory is diagonal theory with self-conjugate primaries we can apply to this situation equation (43) with $i=-b / 2, j=\alpha=\frac{Q}{2}+i P, k=\alpha \pm b / 2$ :

$$
\begin{aligned}
& U_{\mathcal{P}}^{(N)}(\alpha) U_{\mathcal{P}}^{(N)}(-b / 2)= \\
& \left(C_{-b / 2, \alpha}^{\alpha-b / 2} F_{\alpha-b / 2,0}\left[\begin{array}{cc}
-b / 2 & -b / 2 \\
\alpha & \alpha
\end{array}\right]\right)^{N} U_{\mathcal{P}}^{(N)}(\alpha-b / 2) \\
& +\left(C_{-b / 2, \alpha}^{\alpha+b / 2} F_{\alpha+b / 2,0}\left[\begin{array}{cc}
-b / 2 & -b / 2 \\
\alpha & \alpha
\end{array}\right]\right)^{N} U_{\mathcal{P}}^{(N)}(\alpha+b / 2)
\end{aligned}
$$

The necessary three-point functions can be computed using (81) and (82)

$$
\begin{gathered}
C_{-b / 2, \alpha}^{\alpha-b / 2}=C(-b / 2, \alpha, Q-\alpha+b / 2)=1 \\
C_{-b / 2, \alpha}^{\alpha+b / 2}=C(\alpha,-b / 2, Q-\alpha-b / 2)=b^{4} \pi \mu \gamma\left(b^{2}\right) \frac{\Gamma\left(2 \alpha b-b^{2}-1\right) \Gamma(1-2 \alpha b)}{\Gamma\left(2+b^{2}-2 \alpha b\right) \Gamma(2 \alpha b)}
\end{gathered}
$$


The necessary elements of the fusion matrix are computed in $[11,40,44]$ using explicit expression of the conformal blocks through hypergeometric functions. We will write down here final results:

$$
\begin{gathered}
F_{\alpha-b / 2,0}\left[\begin{array}{cc}
-b / 2 & -b / 2 \\
\alpha & \alpha
\end{array}\right]=\frac{\Gamma\left(2 \alpha b-b^{2}\right) \Gamma\left(-1-2 b^{2}\right)}{\Gamma\left(2 \alpha b-2 b^{2}-1\right) \Gamma\left(-b^{2}\right)} \\
F_{\alpha+b / 2,0}\left[\begin{array}{cc}
-b / 2 & -b / 2 \\
\alpha & \alpha
\end{array}\right]=\frac{\Gamma\left(2+b^{2}-2 \alpha b\right) \Gamma\left(-1-2 b^{2}\right)}{\Gamma(1-2 \alpha b) \Gamma\left(-b^{2}\right)}
\end{gathered}
$$

At this point we can continue in two different ways. It is shown in $[11,44]$ that Liouville theory possesses two kinds of boundary states, discrete and continuous families. For permutation branes and defects one expects the same picture. To discover continuous family one treats $U_{\mathcal{P}}^{(N)}(-b / 2)$ as a constant $A$ depending on boundary condition. Doing this and putting (88), (89), (90) and (91) in (87) one receives the following linear equation:

$$
\begin{aligned}
& A U_{\mathcal{P}}^{(N)}(\alpha)=\left(\frac{\Gamma\left(-1-2 b^{2}\right) \Gamma\left(2 \alpha b-b^{2}\right)}{\Gamma\left(-b^{2}\right) \Gamma\left(2 \alpha b-2 b^{2}-1\right)}\right)^{N} U_{\mathcal{P}}^{(N)}(\alpha-b / 2)+ \\
& \left(\frac{\pi \mu \gamma\left(b^{2}\right) b^{4} \Gamma\left(-1-2 b^{2}\right) \Gamma\left(2 \alpha b-b^{2}-1\right)}{\Gamma\left(-b^{2}\right) \Gamma(2 \alpha b)}\right)^{N} U_{\mathcal{P}}^{(N)}(\alpha+b / 2)
\end{aligned}
$$

Using the identity

$$
\Gamma(1+z)=z \Gamma(z)
$$

it is easy to show that (92) can be solved by:

$$
U_{\mathcal{P} s}^{(N)}(\alpha)=2^{1 / 2}\left[\frac{1}{2^{3 / 4} \pi b}\left(\pi \mu \gamma\left(b^{2}\right)\right)^{(Q-2 \alpha) / 2 b} \Gamma(1-b(Q-2 \alpha)) \Gamma\left(-b^{-1}(Q-2 \alpha)\right)\right]^{N} \cosh (2 \pi s(2 \alpha-Q))
$$

where

$$
2 \cosh 2 \pi b s=\frac{A}{b^{2 N}}\left(\frac{\Gamma\left(-b^{2}\right)}{\Gamma\left(-1-2 b^{2}\right)}\right)^{N} \frac{1}{\left(\pi \mu \gamma\left(b^{2}\right)\right)^{N / 2}}
$$

Putting $\alpha=\frac{Q}{2}+i P$ we get

$$
U_{\mathcal{P} s}^{(N)}(P)=2^{1 / 2}\left(\frac{\left[\pi \mu \gamma\left(b^{2}\right)\right]^{-i P / b} \Gamma(1+2 i b P) \Gamma\left(1+\frac{2 i P}{b}\right)}{2^{3 / 4}(2 i \pi P)}\right)^{N} \cos (4 P \pi s)
$$

Let us make the following comments on (96). 
1. Putting $N=1$ we surely recover FZZ branes 1 [11]:

$U_{\mathcal{P} s}^{(1)}(P) \equiv U_{s}^{(F Z Z)}(P)=\frac{2^{-1 / 4}\left[\pi \mu \gamma\left(b^{2}\right)\right]^{-i P / b} \Gamma(1+2 i b P) \Gamma\left(1+\frac{2 i P}{b}\right)}{2 i \pi P} \cos (4 P \pi s)$

2. It is very interesting to note that (96) has similar structure as corresponding solution (3) in the case of rational CFT, in the sense that both have the form $S_{a j}(f(j))^{N}$, where $S_{a j}$ is the matrix of the modular transformation of the single copy, and $f(j)$ is the function which appears in the expression for single copy boundary states.

3. From the expression (84) one concludes that $U_{\mathcal{P}}^{(N)}(P)$ should satisfy

$$
U_{\mathcal{P} s}^{(N)}(P)=(S(P))^{N} U_{\mathcal{P} s}^{(N)}(-P)
$$

Solution (96) obviously satisfies (98).

To obtain discrete family we will treat $U_{\mathcal{P}}^{(N)}(-b / 2)$ as it stands, and again substituting in (87) values of structure constants and elements of fusion matrix (88), (89), (90) and (91), we derive the following non-linear equation:

$$
\begin{aligned}
& U_{\mathcal{P}}^{(N)}(\alpha) U_{\mathcal{P}}^{(N)}(-b / 2)=\left(\frac{\Gamma\left(-1-2 b^{2}\right) \Gamma\left(2 \alpha b-b^{2}\right)}{\Gamma\left(-b^{2}\right) \Gamma\left(2 \alpha b-2 b^{2}-1\right)}\right)^{N} U_{\mathcal{P}}^{(N)}(\alpha-b / 2)+(99) \\
& \left(\frac{\pi \mu b^{4} \gamma\left(b^{2}\right) \Gamma\left(-1-2 b^{2}\right) \Gamma\left(2 \alpha b-b^{2}-1\right)}{\Gamma\left(-b^{2}\right) \Gamma(2 \alpha b)}\right)^{N} U_{\mathcal{P}}^{(N)}(\alpha+b / 2)
\end{aligned}
$$

Equation (99) admits the following two-parameters solution:

$$
U_{\mathcal{P} m, n}^{(N)}(\alpha)=\left(\frac{\left[\pi \mu \gamma\left(b^{2}\right)\right]^{-\alpha / b} \Gamma(1-b(Q-2 \alpha)) \Gamma\left(-b^{-1}(Q-2 \alpha)\right)}{\Gamma(1-b Q) \Gamma\left(-b^{-1} Q\right)}\right)^{N} f_{m, n}(\alpha)
$$

where

$$
f_{m, n}(\alpha)=\frac{\sin \left(\pi m b^{-1}(2 \alpha-Q)\right) \sin (\pi n b(2 \alpha-Q))}{\sin \left(\pi m b^{-1} Q\right) \sin (\pi n b Q)}
$$

and satisfies equation

$$
f_{m, n}(\alpha) f_{m, n}(-b / 2)=f_{m, n}(\alpha-b / 2)+f_{m, n}(\alpha+b / 2)
$$

Putting $\alpha=\frac{Q}{2}+i P$ we get

\footnotetext{
${ }^{1}$ To compare with [11] we changed here slightly normalization, and redefined parameter $s$ there as $2 s$ here.
} 
$U_{\mathcal{P} m, n}^{(N)}(P)=\left(\frac{\left[\pi \mu \gamma\left(b^{2}\right)\right]^{-Q / 2 b}\left[\pi \mu \gamma\left(b^{2}\right)\right]^{-i P / b} \Gamma(1+2 i P b) \Gamma(1+2 i P / b) Q}{\Gamma(1-b Q) \Gamma\left(1-b^{-1} Q\right)(-2 i P)}\right)^{N} f_{m, n}(P)$

where

$$
f_{m, n}(P)=\frac{\sinh \left(2 \pi m P b^{-1}\right) \sinh (2 \pi n b P)}{\sin \left(\pi m b^{-1} Q\right) \sin (\pi n b Q)}
$$

To construct boundary states one should solve additionally the equation (26). The solution is easily seen to be

$\Psi_{\mathcal{P} m, n}^{(N)}(P)=2^{3 / 2}\left(\frac{\left[\pi \mu \gamma\left(b^{2}\right)\right]^{-i P / b} \Gamma(1+2 i P b) \Gamma(1+2 i P / b)}{2^{3 / 4}(2 i \pi P)}\right)^{N} \sinh \left(2 \pi m P b^{-1}\right) \sinh (2 \pi n b P)$

$$
U_{\mathcal{P} m, n}^{(N)}(P)=\frac{\Psi_{\mathcal{P} m, n}^{(N)}(P)}{\Psi_{\mathcal{P} m, n}^{(N)}\left(i \frac{Q}{2}\right)}
$$

For solution (105) we can make similar comments as for solution (96). For $N=1$ we recover ZZ branes:

$$
\begin{aligned}
& \Psi_{\mathcal{P} m, n}^{(1)}(P) \equiv \Psi_{m, n}^{(Z Z)}(P)= \\
& \frac{2^{3 / 4}\left[\pi \mu \gamma\left(b^{2}\right)\right]^{-i P / b} \Gamma(1+2 i P b) \Gamma(1+2 i P / b)}{2 i \pi P} \sinh \left(2 \pi m P b^{-1}\right) \sinh (2 \pi n b P)
\end{aligned}
$$

The solution (105) has the same structure as (3) in the same sense as before, and satisfies the reflection constraint (98).

Having reflection amplitudes (96) and (105) one can write boundary states

$$
\begin{aligned}
|s\rangle_{\mathcal{P}}^{(N)} & \left.=\int U_{\mathcal{P} s}^{(N)}(P)|P\rangle\right\rangle_{\mathcal{P}}^{(N)} d P \\
|m, n\rangle_{\mathcal{P}}^{(N)} & \left.=\int \Psi_{\mathcal{P} m, n}^{(N)}(P)|P\rangle\right\rangle_{\mathcal{P}}^{(N)} d P
\end{aligned}
$$

where $|P\rangle\rangle_{\mathcal{P}}^{(N)}$ are Ishibashi states satisfying (푸). For $N=1$ we identify

$$
\begin{gathered}
\left.|s\rangle_{\mathcal{P}}^{(1)} \equiv|s\rangle^{(F Z Z)}=\int U_{s}^{(F Z Z)}(P)|P\rangle\right\rangle d P \\
\left.|m, n\rangle_{\mathcal{P}}^{(1)} \equiv|m, n\rangle^{(Z Z)}=\int \Psi_{m, n}^{(Z Z)}(P)|P\rangle\right\rangle d P
\end{gathered}
$$

where $|P\rangle\rangle$ are the Ishibashi states satisfying $L_{n}+\bar{L}_{-n}=0$. 
Let us test the solutions (108) and (109) computing the annulus partition function between permutation branes and products of ZZ branes. For simplicity we restrict ourselves to the case of permutation branes on two-fold product $N=2$. The partition function between permutation brane labelled by $s$ and product of two ZZ branes labelled by $\left(m_{1}, n_{1}\right)$ and $\left(m_{2}, n_{2}\right)$ respectively is

$$
\begin{aligned}
& Z_{s,\left(m_{1}, n_{1}\right),\left(m_{2}, n_{2}\right)}=\int U_{\mathcal{P} s}^{(2)}(-P) \Psi_{m_{1}, n_{1}}^{Z Z}(P) \Psi_{m_{2}, n_{2}}^{(Z Z)}(P)\left(\chi_{P}(\tilde{q})\right)^{2} d P= \\
& \int_{P} \frac{2^{1 / 2} \cos (4 P \pi s) \sinh \left(2 \pi m_{1} b P\right) \sinh \left(2 \pi n_{1} P / b\right) \sinh \left(2 \pi m_{2} b P\right) \sinh \left(2 \pi n_{2} P / b\right)}{(\sinh (2 b P) \sinh (2 P / b))^{2}} \chi_{P}\left(\tilde{q}^{2}\right) d P
\end{aligned}
$$

To obtain (112) we used the $\Gamma$-function identity

$$
\Gamma(1+i x) \Gamma(1-i x)=\frac{\pi x}{\sinh (\pi x)}
$$

and the following property of the permutation Ishibashi states

$$
\left\langle\left\langle Q_{1}\right|\left\langle\left\langle Q_{2}\left|\left(\tilde{q}^{1 / 2}\right)^{H}\right| P\right\rangle\right\rangle_{\mathcal{P}}^{(2)}=\chi_{P}\left(\tilde{q}^{2}\right) \delta\left(P-Q_{1}\right) \delta\left(P-Q_{2}\right)\right.
$$

Using identities

$$
\sinh (2 \pi n b P) \sinh \left(2 \pi n^{\prime} b P\right)=\sum_{l=0}^{\min \left(n, n^{\prime}\right)-1} \sinh (2 \pi b P) \sinh \left(2 \pi b\left(n+n^{\prime}-2 l-1\right) P\right)
$$

and

$$
\frac{\sinh (2 \pi n b P)}{\sinh (2 \pi b P)}=\sum_{l=1-n, 2}^{n-1} \exp (2 \pi l b P)
$$

and performing modular transformation (74) we obtain:

$$
\sum_{l_{1}=0}^{Z_{\left(m_{1}, n_{1}\right),\left(m_{2}, n_{2}\right)}=} \sum_{k_{1}=0}^{\min \left(n_{1}, n_{2}\right)-1} \sum_{l=1-\left(n_{1}+n_{2}-2 l_{1}-1\right), 2}^{\min \left(m_{1}, m_{2}\right)-1} \sum_{k=1-\left(m_{1}+m_{2}-2 k_{1}-1\right), 2}^{\left(n_{1}+n_{2}-2 l_{1}-1\right)-1} \chi_{s+i(k / b+l b) / 2}\left(q^{1 / 2}\right)
$$

in agreement with (10). Again using (113) and (114) for the partition function between permutation brane labeled by $(m, n)$ and product of two ZZ branes labeled by $\left(m_{1}, n_{1}\right)$ and $\left(m_{2}, n_{2}\right)$ one obtains:

$$
\begin{aligned}
& Z_{(m, n) ;\left(m_{1}, n_{1}\right),\left(m_{2}, n_{2}\right)}=\int \Psi_{\mathcal{P} m, n}^{(2)}(-P) \Psi_{m_{1}, n_{1}}^{(Z Z)}(P) \Psi_{m_{2}, n_{2}}^{(Z Z)}(P) \chi_{P}\left(\tilde{q}^{2}\right) d P= \\
& \int_{P} \frac{2^{3 / 2} \sinh (2 \pi m b P) \sinh (2 \pi n P / b) \sinh \left(2 \pi m_{1} b P\right) \sinh \left(2 \pi n_{1} P / b\right) \sinh \left(2 \pi m_{2} b P\right) \sinh \left(2 \pi n_{2} P / b\right)}{(\sinh (2 b P) \sinh (2 P / b))^{2}} \\
& \times \chi_{P}\left(\tilde{q}^{2}\right) d P
\end{aligned}
$$


Using identity (115) and modular transformation law for degenerate characters (77) it takes form

$$
\begin{gathered}
Z_{(m, n) ;\left(m_{1}, n_{1}\right),\left(m_{2}, n_{2}\right)}=\sum_{l_{1}=0}^{\min \left(n_{1}, n_{2}\right)-1} \sum_{k_{1}=0}^{\min \left(m_{1}, m_{2}\right)-1} \sum_{l_{2}=0}^{\min \left(n, n_{1}+n_{2}-2 l_{1}-1\right)-1} \\
\sum_{\min \left(m, m_{1}+m_{2}-2 k_{1}-1\right)-1}^{\chi_{m_{1}+m_{2}+m-2 k_{1}-2 k_{2}-2 ; n_{1}+n_{2}+n-2 l_{1}-2 l_{2}-2}\left(q^{1 / 2}\right)}
\end{gathered}
$$

again in agreement with (10). This calculation can be easily generalized to the the case of generic $N$. It shows in particularly that to produce correct formula for annulus partition function between permutation branes and products of ZZ branes the power $N$ in (96) and (105) is really necessary.

\section{Defects in Liouville theory}

Defects in the Liouville theory can be constructed from the permutation branes $U_{\mathcal{P} s}^{(2)}(P)$ and $\Psi_{\mathcal{P} m, n}^{(2)}(P)$ on two-fold product constructed in the previous section using discussed in section 3 folding trick. As we explained in section 3 folding trick involves two steps, left right exchange and hermitian conjugation. Taking into account two-point function of the Liouville theory (79) one concludes that permutations branes reflection amplitude in the process of the hermitian conju-

gation should be divided by the reflection function (78). Dividing (96) and (105) for $N=2$ by the reflection function (78) and using (113) one obtains:

$$
D_{s}(P)=U_{\mathcal{P} s}^{(2)}(P) S(-P)=\frac{\cos (4 P \pi s)}{2 \sinh (2 \pi b P) \sinh (2 P \pi / b)}
$$

and

$$
\mathcal{D}_{m, n}(P)=\Psi_{\mathcal{P} m, n}^{(2)}(P) S(-P)=\frac{\sinh \left(2 \pi m P b^{-1}\right) \sinh (2 \pi n b P)}{\sinh (2 \pi b P) \sinh (2 P \pi / b)}
$$

Now one can define

$$
X_{s}=\int_{P} D_{s}(P) \operatorname{id}_{P \otimes P} d P
$$

and

$$
X_{m, n}=\int_{P} \mathcal{D}_{m, n}(P) \operatorname{id}_{P \otimes P} d P
$$


where $\operatorname{id}_{P \otimes P}$ is the identity operator on the space $R_{\frac{Q}{2}+i P} \otimes R_{\frac{Q}{2}+i P}$. Consider partition function with insertion of two defects parameterized by $\left(m_{1}, n_{1}\right)$ and $\left(m_{2}, n_{2}\right)$

$$
\begin{aligned}
& Z_{\left(m_{1}, n_{1}\right),\left(m_{2}, n_{2}\right)}=\int \mathcal{D}_{m_{1}, n_{1}}(P) \mathcal{D}_{m_{2}, n_{2}}(P) \chi_{P}(\tilde{q}) \chi_{P}(\overline{\tilde{q}}) d P= \\
& \int_{P} \frac{\sinh \left(2 \pi m_{1} b P\right) \sinh \left(2 \pi n_{1} P / b\right) \sinh \left(2 \pi m_{2} b P\right) \sinh \left(2 \pi n_{2} P / b\right)}{(\sinh (2 b P) \sinh (2 P / b))^{2}} \chi_{P}(\tilde{q}) \chi_{P}(\overline{\tilde{q}}) d P
\end{aligned}
$$

Using identities (115) and (116) and performing modular transformation (74) we obtain:

$$
\int \sum_{l_{1}=0}^{Z_{\left(m_{1}, n_{1}\right),\left(m_{2}, n_{2}\right)}} \sum_{k_{1}=0}^{\min \left(n_{1}, n_{2}\right)-1} \sum_{l=1-\left(n_{1}+n_{2}-2 l_{1}-1\right), 2}^{\min \left(m_{1}, m_{2}\right)-1} \sum_{k=1-\left(m_{1}+m_{2}-2 k_{1}-1\right), 2}^{\left(n_{1}+n_{2}-2 l_{1}-1\right)-1} \chi_{P+i(k / b+l b) / 2}(q) \chi_{P}(\bar{q}) d P
$$

in agreement with (49).

Using identities (115) and (116) for fusion of defects with boundaries and with themselves one obtains

$$
\begin{gathered}
X_{m, n}\left|m^{\prime}, n^{\prime}\right\rangle^{(Z Z)}=\sum_{l=0}^{\min \left(n, n^{\prime}\right)-1} \sum_{k=0}^{\min \left(m, m^{\prime}\right)-1}\left|m+m^{\prime}-2 k-1, n+n^{\prime}-2 l-1\right\rangle^{(Z Z)} \\
X_{m, n}|s\rangle^{(F Z Z)}=\sum_{l=1-n, 2}^{n-1} \sum_{k=1-m, 2}^{m-1}|s+i(k / b+l b) / 2\rangle^{(F Z Z)} \\
\left.X_{s}|m, n\rangle^{(Z Z)}=\sum_{l=1-n, 2}^{n-1} \sum_{k=1-m, 2}^{m-1} s+i(k / b+l b) / 2\right\rangle^{(F Z Z)} \\
X_{m, n} X_{m^{\prime}, n^{\prime}}=\sum_{l=0}^{\min \left(n, n^{\prime}\right)-1} \sum_{k=0}^{\min \left(m, m^{\prime}\right)-1} X_{m+m^{\prime}-2 k-1, n+n^{\prime}-2 l-1} \\
X_{m, n} X_{s}=\sum_{l=1-n, 2}^{n-1} \sum_{k=1-m, 2}^{m-1} X_{s+i(k / b+l b) / 2}
\end{gathered}
$$

in agreement with (50) and (51). 


\section{Discussion}

We would like to outline here some directions for future work.

- In this paper we have constructed defects and permutation branes in the Liouville field theory, using the classifying algebra technique. This technique can be used to find defects and permutation branes also in other non-rational models like $S L(2, R), S L(2, R) / U(1)$, Nappi-Witten etc.

- Another important task is to study defects and permutation branes in the Lagrangian approach to the Liouville field theory. We can write a following mixed boundary interaction term

$$
\mu_{B} e^{\alpha \varphi_{1}} e^{\beta \varphi_{2}}
$$

where $\alpha(Q-\alpha)+\beta(Q-\beta)=1$, in the product space of the two Liouville fields $\varphi_{1}$ and $\varphi_{2}$. In the case when $\alpha=\beta$ one has the permutation symmetry. We are tempted to think that parameter $A$, labeling continuous family in (92), should be related to the parameter $\mu_{B}$ in (131) for this case.

- The defect $X_{s_{1}}$ acting on FZZ states $\left|s_{2}\right\rangle^{(F Z Z)}$ produces the state:

$$
\left.X_{s_{1}}\left|s_{2}\right\rangle^{(F Z Z)}=\int \frac{\cos \left(4 P \pi s_{1}\right)}{2 \sinh (2 \pi b P) \sinh (2 P \pi / b)} U_{s_{2}}^{(F Z Z)}(P)|P\rangle\right\rangle d P
$$

The interpretation of this state at the moment is not clear. It would be interesting to understand this state in the matrix model approach $[24,25]$.

\section{Acknowledgements}

I am grateful to Ingo Runkel and Christoph Schweigert for useful discussions and comments on the paper.

Author received partial support from the Collaborative Research Centre 676

"Particles, Strings and the Early Universe - the Structure of Matter and SpaceTime".

\section{References}

[1] C. Bachas, J. de Boer, R. Dijkgraaf and H. Ooguri, "Permeable conformal walls and holography," JHEP 0206 (2002) 027 [arXiv:hep-th/0111210]. 
[2] C. Bachas and I. Brunner, "Fusion of conformal interfaces," JHEP 0802 (2008) 085 arXiv:0712.0076 [hep-th]].

[3] R. E. Behrend, P. A. Pearce, V. B. Petkova and J. B. Zuber, "Boundary conditions in rational conformal field theories," Nucl. Phys. B 570 (2000) 525 [Nucl. Phys. B 579 (2000) 707] arXiv:hep-th/9908036].

[4] E. Braaten, T. Curtright and C. B. Thorn, "An Exact Operator Solution Of The Quantum Liouville Field Theory," Annals Phys. 147 (1983) 365.

[5] E. Braaten, T. Curtright, G. Ghandour and C. B. Thorn, "Nonperturbative Weak Coupling Analysis Of The Quantum Liouville Field Theory," Annals Phys. 153 (1984) 147.

[6] J. L. Cardy, "Boundary Conditions, Fusion Rules And The Verlinde Formula," Nucl. Phys. B 324 (1989) 581.

[7] J. L. Cardy and D. C. Lewellen, "Bulk and boundary operators in conformal field theory," Phys. Lett. B 259 (1991) 274.

[8] T. L. Curtright and C. B. Thorn, "Conformally Invariant Quantization Of The Liouville Theory," Phys. Rev. Lett. 48 (1982) 1309 [Erratum-ibid. 48 (1982) 1768].

[9] H. Dorn and H. J. Otto, "Two and three point functions in Liouville theory," Nucl. Phys. B 429 (1994) 375 arXiv:hep-th/9403141.

[10] V. S. Dotsenko and V. A. Fateev, "Four Point Correlation Functions And The Operator Algebra In The Two-Dimensional Conformal Invariant Theories With The Central Charge $C<1$," Nucl. Phys. B 251 (1985) 691.

[11] V. Fateev, A. B. Zamolodchikov and A. B. Zamolodchikov, "Boundary Liouville field theory. I: Boundary state and boundary two-point arXiv:hep-th/0001012.

[12] J. Frohlich, J. Fuchs, I. Runkel and C. Schweigert, "Kramers-Wannier duality from conformal defects," Phys. Rev. Lett. 93 (2004) 070601 arXiv:cond-mat/0404051]. 
[13] J. Frohlich, J. Fuchs, I. Runkel and C. Schweigert, "Duality and defects in rational conformal field theory," Nucl. Phys. B 763 (2007) 354 arXiv:hep-th/0607247.

[14] J. Fuchs, I. Runkel and C. Schweigert, "Boundaries, defects and Frobenius algebras," Fortsch. Phys. 51 (2003) 850 [Annales Henri Poincare 4 (2003) S175] arXiv:hep-th/0302200.

[15] J. Fuchs, I. Runkel and C. Schweigert, "TFT construction of RCFT correlators. I: Partition functions," Nucl. Phys. B 646 (2002) 353 arXiv:hep-th/0204148.

[16] J. Fuchs, M. R. Gaberdiel, I. Runkel and C. Schweigert, "Topological defects for the free boson CFT," J. Phys. A 40 (2007) 11403 arXiv:0705.3129 [hepth]].

[17] J. Fuchs, C. Schweigert and K. Waldorf, "Bi-branes: Target space geometry for world sheet topological defects," J. Geom. Phys. 58 (2008) 576 arXiv:hep-th/0703145.

[18] M. R. Gaberdiel and S. Schafer-Nameki, "D-branes in an asymmetric orbifold," Nucl. Phys. B 654 (2003) 177 arXiv:hep-th/0210137.

[19] A. Giveon, D. Kutasov and A. Schwimmer, "Comments on D-branes in AdS(3)," Nucl. Phys. B 615 (2001) 133 arXiv:hep-th/0106005.

[20] K. Graham and G. M. T. Watts, "Defect lines and boundary flows," JHEP 0404 (2004) 019 arXiv:hep-th/0306167.

[21] V. G. Kac, "Infinite dimensional Lie algebras," Cambridge, UK: Univ. Pr. (1990) $400 p$

[22] P. Lee, H. Ooguri and J. w. Park, "Boundary states for AdS(2) branes in AdS(3)," Nucl. Phys. B 632 (2002) 283 arXiv:hep-th/0112188.

[23] D. C. Lewellen, "Sewing constraints for conformal field theories on surfaces with boundaries," Nucl. Phys. B 372 (1992) 654.

[24] E. J. Martinec, "The annular report on non-critical string theory," arXiv:hep-th/0305148. 
[25] E. J. Martinec, "Matrix models and 2D string theory," arXiv:hep-th/0410136.

[26] G. W. Moore and N. Seiberg, "Classical and Quantum Conformal Field Theory," Commun. Math. Phys. 123 (1989) 177.

[27] M. Oshikawa and I. Affleck, "Boundary conformal field theory approach to the critical two-dimensional Ising model with a defect line," Nucl. Phys. B 495 (1997) 533 [arXiv:cond-mat/9612187].

[28] V. B. Petkova and J. B. Zuber, "Generalised twisted partition functions," Phys. Lett. B 504 (2001) 157 arXiv:hep-th/0011021.

[29] V. B. Petkova and J. B. Zuber, "The many faces of Ocneanu cells," Nucl. Phys. B 603 (2001) 449 [arXiv:hep-th/0101151].

[30] B. Ponsot, V. Schomerus and J. Teschner, "Branes in the Euclidean AdS(3)," JHEP 0202 (2002) 016 [arXiv:hep-th/0112198].

[31] G. Pradisi, A. Sagnotti and Y. S. Stanev, "Completeness Conditions for Boundary Operators in 2D Conformal Field Theory," Phys. Lett. B 381 (1996) 97 arXiv:hep-th/9603097.

[32] A. Recknagel, "Permutation branes," JHEP 0304 (2003) 041 arXiv:hep-th/0208119.

[33] I. Runkel, "Boundary structure constants for the A-series Virasoro minimal models," Nucl. Phys. B 549 (1999) 563 arXiv:hep-th/9811178.

[34] I. Runkel, "Structure constants for the D-series Virasoro minimal models," Nucl. Phys. B 579 (2000) 561 arXiv:hep-th/9908046.

[35] I. Runkel, "Perturbed Defects and T-Systems in Conformal Field Theory," J. Phys. A 41 (2008) 105401 [arXiv:0711.0102 [hep-th]].

[36] I. Runkel and R. R. Suszek, "Gerbe-holonomy for surfaces with defect networks," arXiv:0808.1419 [hep-th].

[37] G. Sarkissian and M. Zamaklar, "Symmetry breaking, permutation D-branes on group manifolds: Boundary states and geometric description," Nucl. Phys. B 696 (2004) 66 arXiv:hep-th/0312215. 
[38] G. Sarkissian and C. Schweigert, "Some remarks on defects and T-duality," arXiv:0810.3159 [hep-th].

[39] C. Schweigert and E. Tsouchnika, "Kramers-Wannier dualities for WZW theories and minimal models," arXiv:0710.0783 [hep-th].

[40] J. Teschner, "On the Liouville three point function," Phys. Lett. B 363 (1995) 65 arXiv:hep-th/9507109|.

[41] J. Teschner, "Remarks on Liouville theory with boundary," arXiv:hep-th/0009138.

[42] J. Teschner, "Liouville theory revisited," Class. Quant. Grav. 18 (2001) R153 arXiv:hep-th/0104158.

[43] E. Wong and I. Affleck, "Tunneling in quantum wires: A Boundary conformal field theory approach," Nucl. Phys. B 417 (1994) 403.

[44] A. B. Zamolodchikov and A. B. Zamolodchikov, "Liouville field theory on a pseudosphere," arXiv:hep-th/0101152.

[45] A. B. Zamolodchikov and A. B. Zamolodchikov, "Structure constants and conformal bootstrap in Liouville field theory," Nucl. Phys. B 477 (1996) 577 arXiv:hep-th/9506136. 